\title{
Impacts des dépôts atmosphériques acides sur I'eau des sols forestiers
}

\author{
Elisabeth Graf Pannatier \\ Anne Thimonier \\ Maria Schmitt \\ Peter Waldner \\ Lorenz Walthert
}

Institut fédéral de recherches sur la forêt, la neige et le paysage $(\mathrm{CH})^{*}$ Institut fédéral de recherches sur la forêt, la neige et le paysage $(\mathrm{CH})$ Institut fédéral de recherches sur la forêt, la neige et le paysage $(\mathrm{CH})$ Institut fédéral de recherches sur la forêt, la neige et le paysage $(\mathrm{CH})$ Institut fédéral de recherches sur la forêt, la neige et le paysage $(\mathrm{CH})$

\section{Impacts of atmospheric acid deposition on soil solutions in forests}

\begin{abstract}
After a massive input of acidifying components on the environment in the middle of the $20^{\text {th }}$ century, atmospheric acid deposition has decreased as a result of sulphur emission reduction. The continuous acid input might affect the chemistry of soils and drainage waters and accelerate soil acidification. In the framework of the Swiss Long-Term Forest Ecosystem Research (LWF), we examined whether acid deposition has continued to decline in the last ten years in different forest ecosystems and how the chemistry of soil water reacted to the improvement in air quality. Acid deposition decreased significantly at only three out of the nine study sites. Sulphur deposition declined at all sites, but due to the relatively low sulphur load compared to nitrogen deposition, it did not contribute to decrease acid deposition. Chemistry of soil solution remained quite constant since the beginning of the measurements about ten years ago. We did not observe any acidification of soil solution in six out of eight sites. In contrast, changes in soil solution chemistry at two sites showed a rapid acidification. At three sites, the deeper soil layer released large amount of sulphate coupled with base cations, which likely contributed to deplete the soil in nutrients. The analysis of the base saturation in 1039 soil profiles across Switzerland shows a high risk of relatively fast acidification of soil solution in almost $20 \%$ of sites.
\end{abstract}

Keywords: acid deposition, soil acidification, soil solution, ICP Forests, LWF doi: $10.3188 /$ szf. 2012.0363

*Zürcherstrasse 111, CH-8903 Birmensdorf, courriel elisabeth.pannatier@wsl.ch

$\mathrm{L}$ 'apport continu d'acides d'origine atmosphérique durant des décennies peut modifier la chimie des sols et des eaux de percolation dans les écosystèmes forestiers et accélérer leur acidification naturelle (Hildebrand et al 1996). Dans les sols acides en particulier, les apports de substances acidifiantes augmentent la mobilité de l'aluminium (Al) dans l'eau du sol, toxique à haute concentration pour les espèces végétales sensibles. Ils entraînent également un lessivage accru des cations basiques $\left(\mathrm{BC}=\mathrm{Ca}^{2+}, \mathrm{Mg}^{2+}, \mathrm{K}^{+}\right)$, éléments essentiels pour la nutrition des plantes. Un déficit en nutriments peut affecter la production de la biomasse, la santé des arbres ainsi que leur sensibilité aux maladies (voir aussi Thimonier et al 2012, ce volume).

Les émissions de dioxyde de soufre $\left(\mathrm{SO}_{2}\right)$ et d'azote sous forme réduite $\left(\mathrm{NH}_{3}\right)$ ou oxydée $\left(\mathrm{NO}_{\mathrm{x}}\right)$ sont à l'origine des dépôts acides. Une fois dans l'atmosphère, ces gaz forment des polluants secondaires comme l'acide nitrique et l'acide sulfurique. Ces émissions acidifiantes ont augmenté massivement depuis le milieu du XXe siècle en Europe (Schöpp et al 2003). Après un pic dans les années 1980, les émissions de
$\mathrm{SO}_{2}$ ont diminué considérablement entre 1985 et 1995, grâce aux mesures de protection de l'air. Les émissions d'azote ont également diminué depuis 1990, mais elles restent encore à un niveau élevé. Le recul des émissions de substances acidifiantes a conduit à une diminution des dépôts atmosphériques acides. En Suisse par exemple, une nette augmentation du pH a été mesurée depuis 1985 dans les eaux de pluie à deux stations de mesure (Payerne et Dübendorf) du réseau NABEL (OFEV 2009).

Les écosystèmes aquatiques et terrestres réagissent de manière différenciée à cette amélioration de la qualité de l'air. Plusieurs études ont montré une diminution de l'acidité dans les rivières et les lacs en Europe dans les années 1990 (Stoddard et al 1999, Evans et al 2001). Mais d'autres travaux n'ont noté aucune amélioration de la qualité des eaux de surface en Allemagne, à cause de la désorption de sulfate emmagasiné dans les sols pendant les années de fortes émissions de soufre (Alewell et al 2000, Armbruster et al 2003). Le sulfate relâché est accompagné de cations basiques, contribuant ainsi à appauvrir les sols. Les apports chroniques d'azote, et 
d'ammonium en particulier, conduisent également à l'acidification des sols. Le nitrate, lorsqu'il excède les besoins nutritionnels des plantes et des microbes, entraîne avec lui des cations basiques dans les eaux d'infiltration. Plusieurs études ont rapporté une augmentation des pertes de cations basiques dans plusieurs bassins versants en Europe (Alewell et al 2001) et en Amérique du Nord (Watmough \& Dillon 2004). L'appauvrissement des sols en cations basiques, qu'il soit causé par une diminution des apports atmosphériques de ces éléments ou par la présence de sulfate ou de nitrate dans les eaux de drainage, est considéré comme un processus majeur causant l'acidification des sols malgré la forte réduction des émissions de soufre. Un indicateur souvent utilisé pour évaluer les risques liés à l'acidification de l'eau du sol et la toxicité de l'aluminium pour les racines des plantes est le rapport $\mathrm{BC} / \mathrm{Al}$, soit le rapport molaire entre les cations basiques et l'aluminium total dissous dans l'eau du sol (Sverdrup \& Warfvinge 1993). Une diminution du rapport $\mathrm{BC} / \mathrm{Al}$ au cours du temps reflète un processus d'acidification de l'eau du sol (Blaser et al 1999).

Les mesures effectuées dans le cadre du projet de recherches à long terme sur les écosystèmes forestiers $(\mathrm{LWF})^{1}$ en Suisse nous permettent d'évaluer la réponse de la qualité des eaux du sol depuis la fin des années 1990, suite aux réductions d'émissions de soufre et d'azote. Les processus menant à des modifications de la composition chimique de la phase solide du sol (acidification par exemple) sont lents. C'est pourquoi nous mesurons la composition chimique de l'eau du sol (dénommée «solution du sol») car elle réagit de manière beaucoup plus sensible que les sols

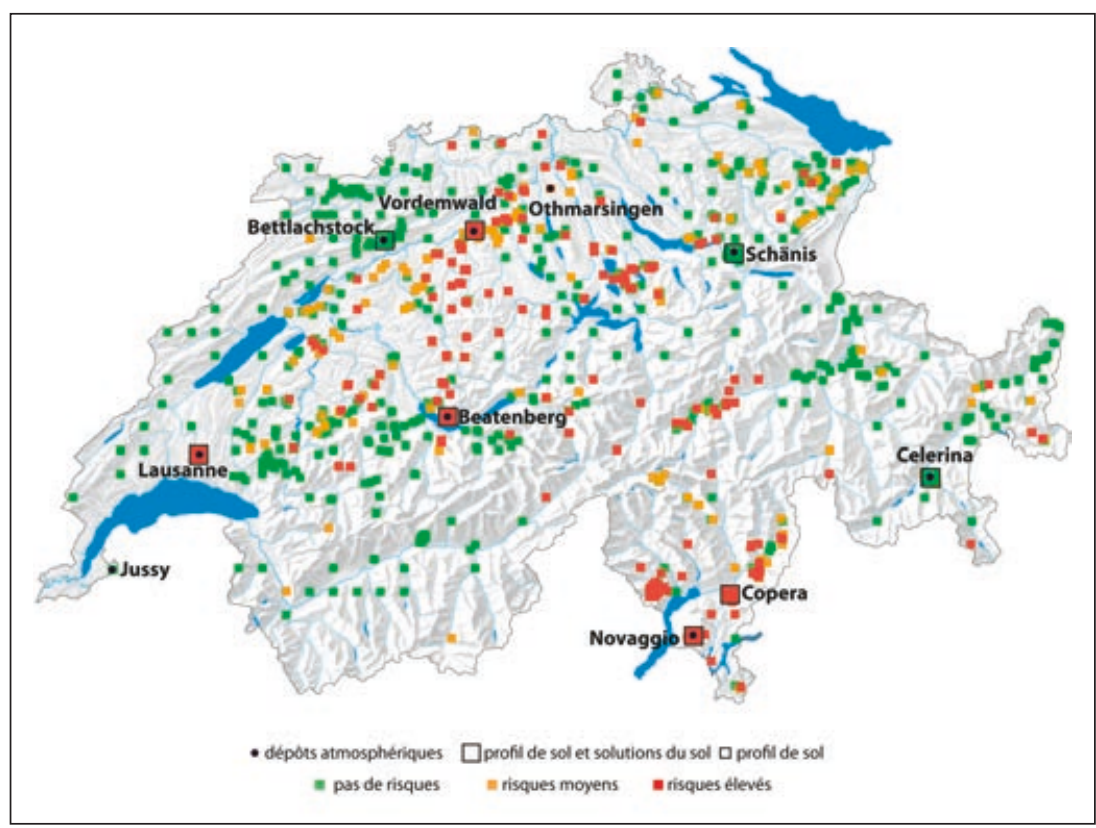

Fig. 1 Localisation des sites d'observation à long terme (LWF et Copera) et des 1039 profils de sol (0-80 cm de profondeur). Les couleurs indiquent le risque d'acidification (diminution probable du rapport BC/AI dans l'eau du sol, voir chapitre «Distribution des sols à complexe désaturé»). Risque élevé: taux de saturation TS moyen $<15 \%$. Risque modéré: TS minimum $<15 \%$ mais TS moyen $\geq 15 \%$. Aucun risque: TS minimum et TS moyen $\geq 15 \%$. aux changements de l'environnement. La qualité chimique de l'eau dépend de plusieurs facteurs: des apports d'éléments dissous provenant de la décomposition de la matière organique, des pluviolessivats ou de l'altération des minéraux et de la roche, de la perte d'éléments par drainage, de l'absorption des racines et de l'échanges d'éléments avec le complexe absorbant (argilo-humique) du sol. L'influence des dépôts atmosphériques sur la composition chimique de l'eau du sol est par conséquent surtout visible dans les sols où les flux d'éléments provenant d'autres sources sont plus petits ou comparables.

Les résultats obtenus sur les sites LWF nous permettent d'élaborer des prédictions pour d'autres sites forestiers où seules les données de sol sont disponibles. Nous disposons aujourd'hui des données chimiques de 1039 profils de sol répartis dans toute la Suisse.

L'objectif de cette publication est de répondre aux trois questions suivantes; comment les dépôts atmosphériques acides ont-ils évolué sur les sites LWF depuis le début des mesures (fin des années 1990)? Comment a évolué la composition chimique de la solution du sol, notamment les concentrations et les flux de sulfate et les rapports BC/Al, sur les sites LWF depuis la fin des années 1990? Où sont situés les sols forestiers dans lesquels le risque d'acidification de l'eau du sol (diminution de BC/Al) est élevé dans les prochaines décennies?

Pour en savoir davantage sur les méthodes et les résultats obtenus sur les sites LWF, le lecteur peut se référer au travail de Graf Pannatier et al (2011).

\section{Matériel et méthodes}

\section{Sites}

Les données présentées dans cet article proviennent du projet de recherche LWF. Les mesures de dépôts atmosphériques proviennent de neuf stations et celles de la solution du sol de sept sites LWF (figure 1). Leurs caractéristiques (altitude, essence forestière principale, type de sol) sont résumées dans le tableau 1. L'installation des sites s'est échelonnée sur plusieurs années. Les échantillonnages des dépôts ont commencé entre 1995 et 2000, et ceux des solutions du sol entre 1999 et 2001 . Nous présentons dans cet article les données jusqu'en 2009, ce qui représente entre 9 et 15 ans de mesures. Les analyses des solutions du sol collectées lors de la première année ont été écartées car l'installation des lysimètres a éventuellement perturbé le sol. L'eau du sol a été également analysée depuis 1987 dans une forêt de châtaigniers à Copera au Tessin, dans le cadre d'un autre projet (Blaser et al 1999, Graf Pannatier et al 2005). Cette série constitue l'enregistrement le plus

1 www.lwf.ch (31.07.2012) 


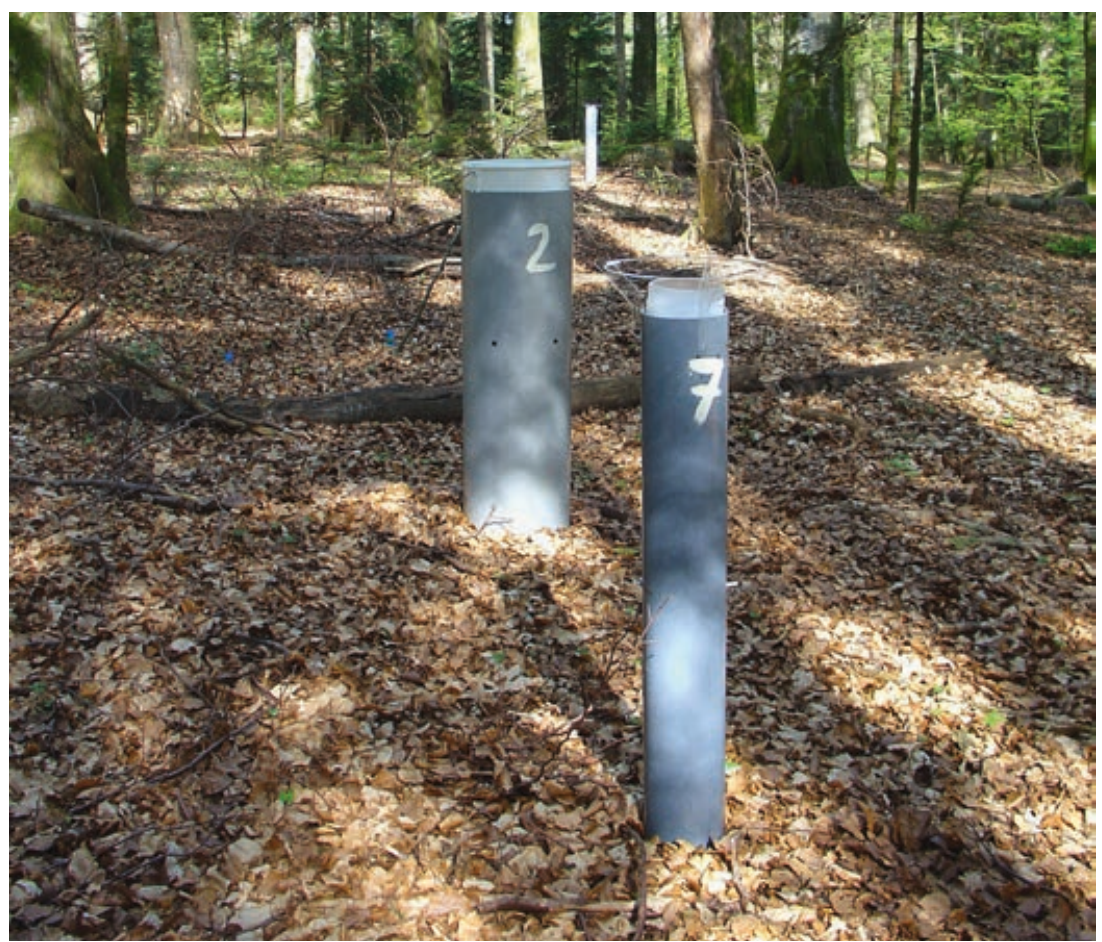

Fig. 2 Collecteurs de pluie et de neige à Lausanne.

long disponible en Suisse. Seules les données depuis 2000 sont présentées dans cet article, afin de pouvoir les comparer aux autres sites. La solution du sol est échantillonnée dans des sols très différents. De manière succincte, on peut les classer en deux catégories: 1) les sols qui se sont développés à partir d'un substrat acide comme à Celerina, Novaggio, Copera, Beatenberg, 2) et ceux qui se sont développés à partir de substrats contenant du calcaire comme à Bettlachstock, Schänis, Vordemwald et Lausanne. L'épaisseur du sol décarbonaté diffère d'un site à l'autre. La limite supérieure de calcaire se situe entre $5 \mathrm{~cm}$ de profondeur à Bettlachstock et plus de $450 \mathrm{~cm}$ de profondeur à Vordemwald (tableau 1). Les caractéristiques physico-chimiques des sols des sites LWF sont décrites dans Walthert et al (2003). Les 1039 profils de sol forestier présentés dans cet article sont répartis dans toute la Suisse (figure 1) et ont été échantillonnés dans différents projets menés à l'Institut fédéral de recherches sur la forêt, la neige et le paysage (WSL).

\section{Dépôts atmosphériques acides}

Les précipitations (hors couvert) et les pluviolessivats (sous couvert) ont été échantillonnés tous les 14 jours, sauf en hiver à Bettlachstock et à Schänis (échantillonnage mensuel). Les précipitations ont été recueillies à l'aide de trois collecteurs hors couvert et seize sous couvert (figures 2 et 3). En hiver, dans les régions en altitude, quatre seaux ont été installés sous couvert pour échantillonner la neige. Les méthodes d'échantillonnage sont décrites en détails dans Thimonier et al (2005). Les flux d'éléments dans les précipitations hors et sous couvert ont été calculés en multipliant la quantité de précipitations avec les concentrations mesurées pendant chaque période d'échantillonnage et additionnés pour obtenir les flux annuels.

Les dépôts atmosphériques totaux comportent les apports humides (pluie, neige) et secs (gaz, particules). Les précipitations hors couvert contiennent les dépôts atmosphériques humides, alors que les pluviolessivats incluent les apports secs retenus par la couronne des arbres et les dépôts humides. Pour estimer les dépôts secs, il faut tenir compte des échanges d'azote, de protons et de cations avec le feuillage en utilisant un modèle («canopy budget

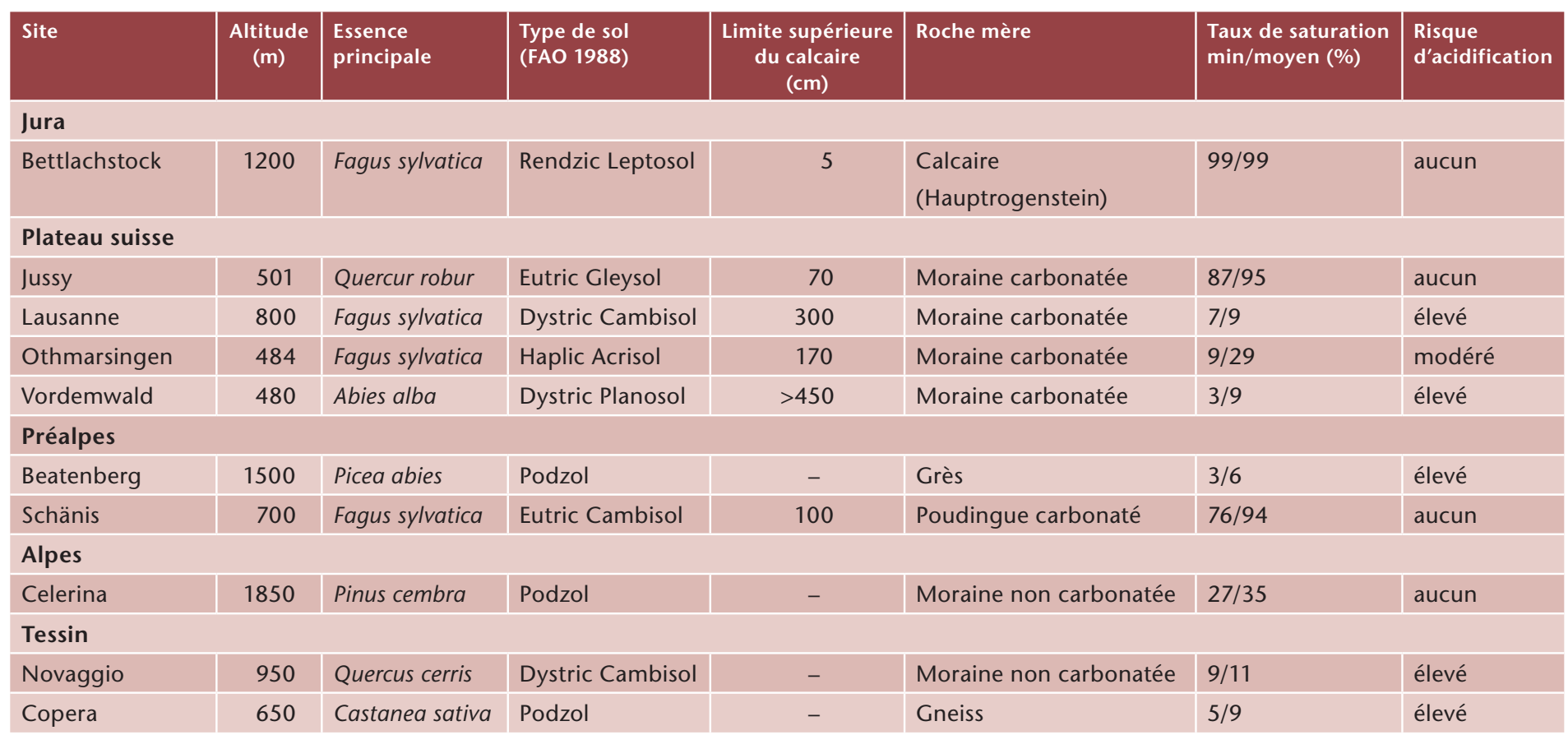

Tab. 1 Description des stations LWF analysées dans cette étude et de Copera: altitude, essence forestière dominante, type de sol, taux de saturation du sol minimum et moyen entre 0 et $80 \mathrm{~cm}$ de profondeur, et risque d'acidification de l'eau du sol. - indique que la roche mère ne contient pas de carbonates. 
Fig. 3 Collecteur de pluie (a), installation d'un lysimètre soustension avec bougie en céramique (b) et échantillons de solution $d u$ sol (c).
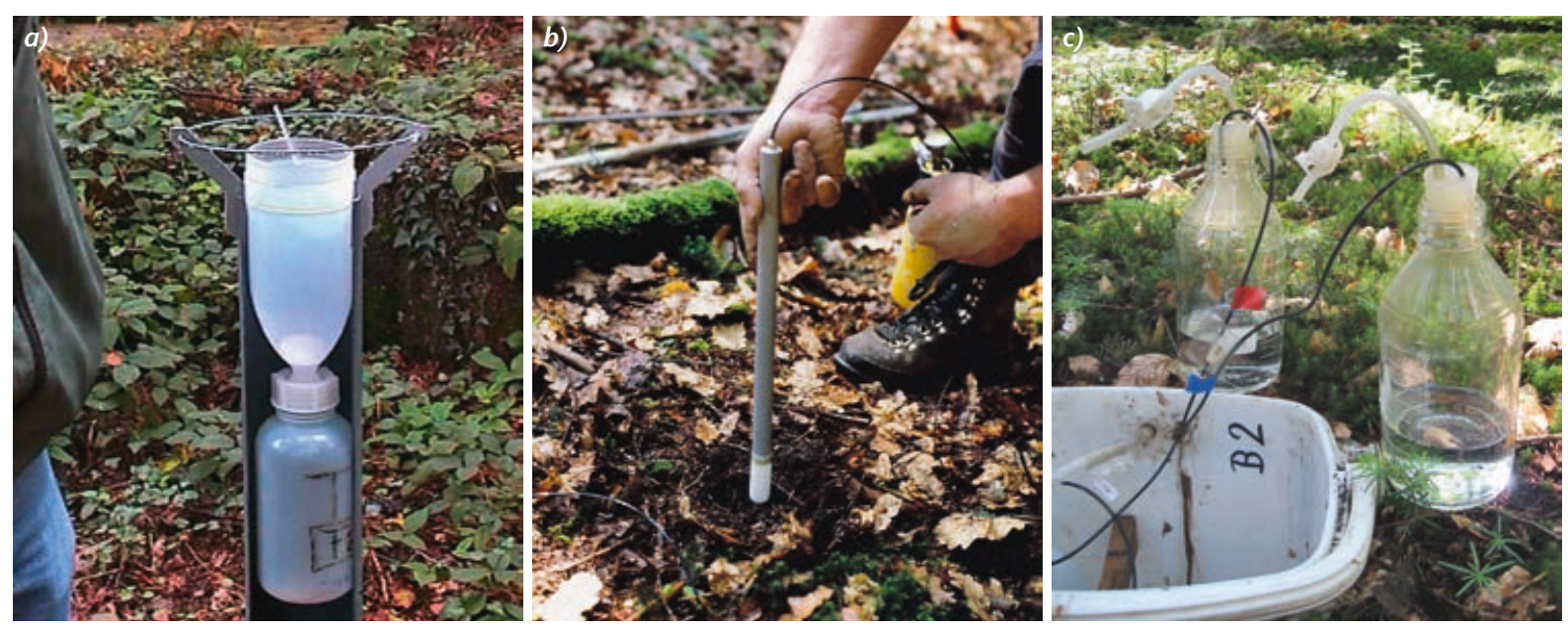

model» développé par Ulrich [1983] et synthétisé par de Vries et al [2001]²).

Les apports atmosphériques acides sont définis comme la somme des dépôts atmosphériques totaux de soufre (S), d'azote $(\mathrm{N})$ et de chlore $\left(\mathrm{Cl}^{-}\right)$moins les dépôts de cations basiques $\left(\mathrm{BC}=\mathrm{Ca}^{2+}+\mathrm{Mg}^{2+}+\mathrm{K}^{+}\right)$ en équivalents (UNECE 2004). Une correction a été réalisée pour soustraire les retombées atmosphériques d'origine marine (UNECE 2004). La méthode de calcul est décrite dans Graf Pannatier et al (2011).

\section{Solutions du sol}

Parallèlement aux précipitations, l'eau du sol a été échantillonnée à l'aide de plaques gravitaires installées de manière permanente sous la litière $(0 \mathrm{~cm})$ et par succion $(-500 \mathrm{hPa})$, à l'aide de lysimètres avec bougie en céramique à $15 \mathrm{~cm}, 50 \mathrm{~cm}$ et $80 \mathrm{~cm}$ de profondeur (figure 3). Tous les échantillons de précipitations et de solutions du sol ont été envoyés au laboratoire, où ils ont été filtrés. Les paramètres suivants ont été ensuite analysés: $\mathrm{pH}$, conductivité électrique, carbone et azote organique dissous, alcalinité, ammonium $\left(\mathrm{NH}_{4}{ }^{+}\right)$, aluminium total dissous (Al), calcium $\left(\mathrm{Ca}^{2+}\right)$, magnésium $\left(\mathrm{Mg}^{2+}\right)$, potassium $\left(\mathrm{K}^{+}\right)$, sodium $\left(\mathrm{Na}^{+}\right)$, fer total dissous $(\mathrm{Fe})$, nitrate $\left(\mathrm{NO}_{3}{ }^{-}\right.$ ), sulfate $\left(\mathrm{SO}_{4}^{2-}\right)$ et chlore $\left(\mathrm{Cl}^{-}\right)$.

Afin de comparer les quantités de soufre arrivant et quittant l'écosystème forestier au cours du temps, nous avons comparé les apports annuels de sulfate provenant des pluviolessivats aux flux de sulfate quittant la litière $(0 \mathrm{~cm})$ et l'espace racinaire à $80 \mathrm{~cm}$ de profondeur depuis le début des mesures. Les flux de sulfate dans le sol (sous la litière et à $80 \mathrm{~cm}$ de profondeur) ont été calculés en multipliant les concentrations dans la solution du sol avec les flux d'eau pendant chaque période d'échantillonnage et additionnés pour obtenir les flux annuels. Les flux d'eau à travers le sol ont été modélisés à l'aide du modèle Coupmodel (Jansson \& Karlberg 2004) et validés en utilisant les mesures de potentiel matriciel (ou force de succion), mesuré tous les 14 jours (Graf Pannatier et al 2011). Pour suivre l'acidification de l'eau du sol au cours du temps, nous avons observé l'évolution temporelle du rapport BC/ Al dans l'eau du sol (Blaser et al 1999).

\section{Sols}

Les échantillons de sol ont été prélevés dans les horizons de 1039 profils répartis dans toute la Suisse. Les profils ont été creusés jusqu'à l'horizon BC ou C si possible, c'est-à-dire jusqu'à la roche mère peu altérée. Les cations échangeables ( $\mathrm{Na}, \mathrm{K}, \mathrm{Mg}$, $\mathrm{Ca}, \mathrm{Mn}$, $\mathrm{Al}, \mathrm{Fe}) \mathrm{du}$ complexe argilo-humique ont été extraits avec $1 \mathrm{M} \mathrm{NH}_{4} \mathrm{Cl}$ pendant une heure, et leurs concentrations ont été mesurées par spectrométrie d'émission optique (ICP-OES). La capacité d'échange effective du complexe absorbant (CEC $\mathrm{Cff}_{\mathrm{f}}$ ) a été calculée en additionnant les concentrations (en $\mathrm{mmol}_{\mathrm{c}} / \mathrm{kg}$ de terre fine) de $\mathrm{Na}, \mathrm{K}, \mathrm{Mg}$, Ca, Mn, $\mathrm{Al}$, Fe et de protons échangeables. Le taux de saturation (TS en \%) est défini comme la proportion de cations échangeables

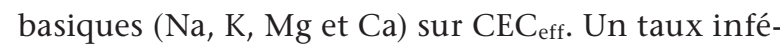
rieur à $15 \%$ est considéré comme critique (Ulrich 1991, Blaser et al 2008) car des apports d'acides, quelles que soient leurs origines, peuvent mener à des conditions très acides dans la solution du sol, causant une forte augmentation des concentrations d'aluminium et une diminution du rapport BC/Al. Nous avons calculé le TS moyen et minimum entre la surface du sol (à partir du premier horizon minéral) et $80 \mathrm{~cm}$ de profondeur. Afin d'identifier les sols dans lesquels il y a un risque d'acidification relativement rapide de l'eau du sol, c'est-à-dire visible en l'espace de 10 ans par une augmentation de la proportion d'aluminium dissous par rapport à celle de cations basiques, nous avons classé les sols en trois catégories de risque: 1 ) risque élevé (TS moyen $<15 \%$ ), 2 ) risque moyen (TS minimum $<15 \%$ mais TS moyen $>15 \%$ ) et 3 ) aucun risque (TS moyen et TS $\min >15 \%$ ).

\section{Analyse des tendances temporelles}

Afin de déterminer les tendances monotones (soit positives ou négatives) dans les séries tempo-

\footnotetext{
2 www.icp-forests.org $\rightarrow$ Publications $\rightarrow$ ICP forest technical reports $\rightarrow$ Technical report 2001 level II (14.8.2012).
} 

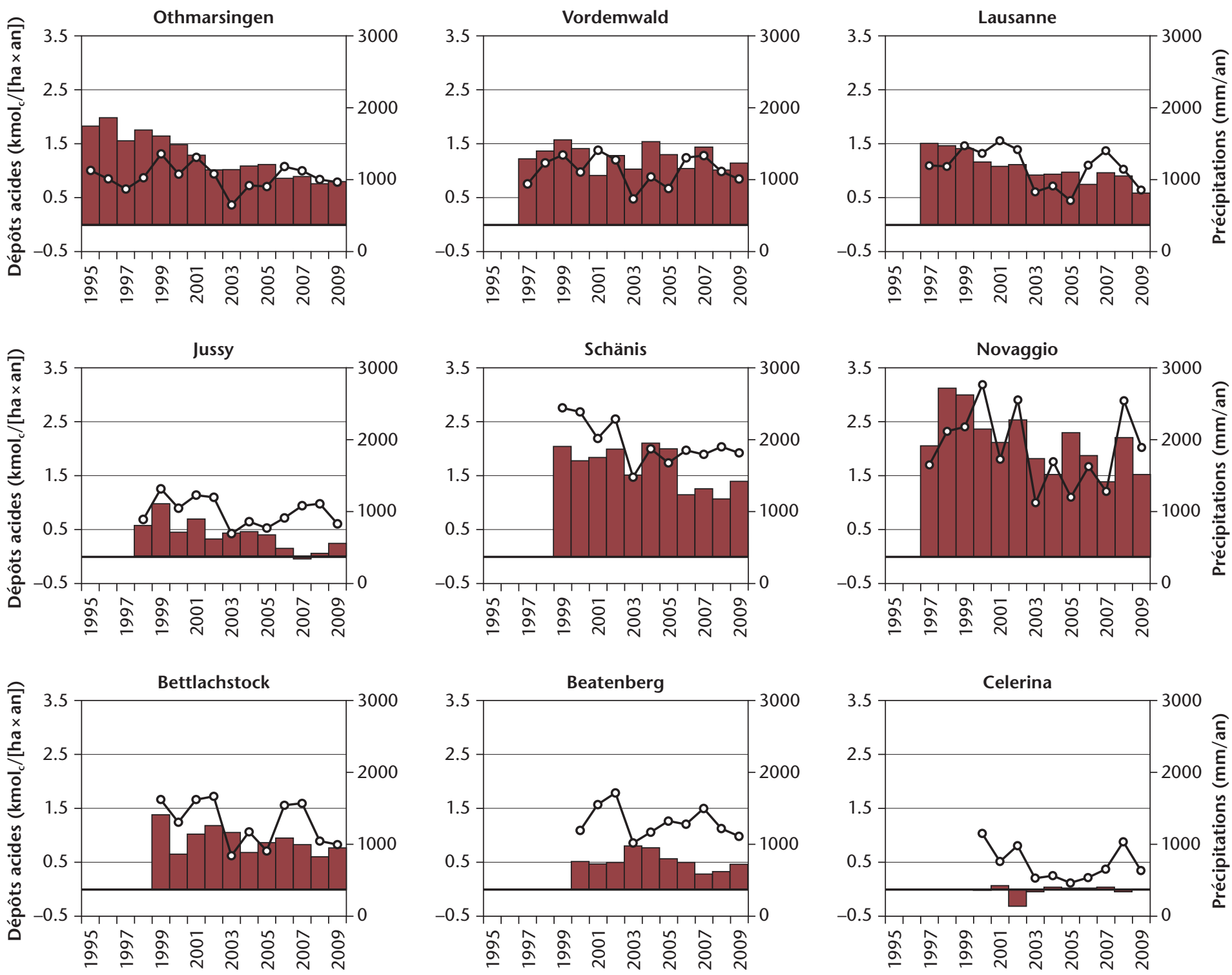

Fig. 4 Séries temporelles des dépôts atmosphériques acides et des précipitations depuis 1995 (ou plus tard) jusqu'en 2009.

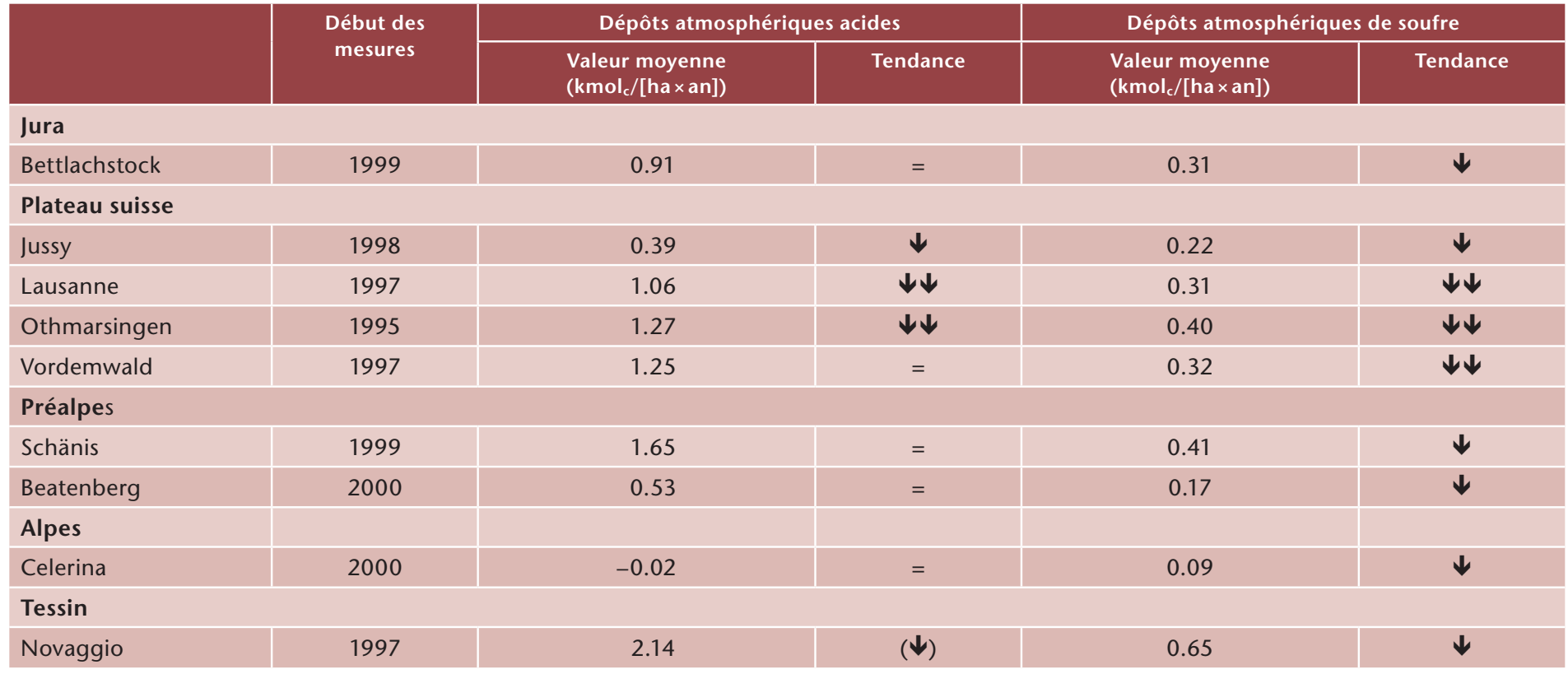

Tab. 2 Tendances temporelles des dépôts atmosphériques acides et de soufre corrigés pour les apports d'origine marine depuis 1995 (ou plus tard) jusqu'en 2009. = pas de tendance; $(\downarrow)$ diminution peu significative $(p<0.05)$; $\downarrow$ diminution significative $(p<0.01)$, $\downarrow$ diminution très significative $(p<0.001)$. 
relles de solution du sol, nous avons utilisé le test saisonnier non paramétrique de Mann-Kendall (Hirsch \& Slack 1984). Nous avons appliqué le test partiel qui permet de soustraire l'effet de dilution ou de concentration causé par différentes teneurs en eau dans le sol (Libiseller \& Grimvall 2002). Pour les dépôts atmosphériques, nous avons utilisé le même test, mais sans saisonnalité avec des valeurs annuelles. Le test partiel permet également de soustraire l'effet des précipitations et de mettre en évidence uniquement l'effet des réductions des émissions. Tous les tests de Mann-Kendall ont été effectués avec le programme MULTMK/PARTMK ${ }^{3}$. Seuls les résultats avec une probabilité $\mathrm{p}<0.05$ ont été considérés comme significatifs.

\section{Résultats et discussion}

\section{Evolution temporelle des dépôts atmosphériques acides}

Les apports atmosphériques acides varient de 0 à $2 \mathrm{kmol}_{\mathrm{c}} /($ ha $\times \mathrm{an})$. Il n'y a pratiquement aucune retombée acide à Celerina dans les Grisons, alors que les sites à Novaggio au Tessin et à Schänis dans les Préalpes reçoivent des apports élevés (figure 4). Ces derniers s'expliquent par la pluviosité importante de ces régions et par la pollution atmosphérique causée par les activités humaines (industrie, transport, agriculture intensive). Les sites du Plateau suisse (Othmarsingen, Vordemwald, Lausanne, Jussy) reçoivent des dépôts acides en quantité modérée, voire faible à Jussy. Les apports atmosphériques acides mesurés ces dix dernières années sur les sites LWF sont inférieurs à ceux estimés à la fin des années 1980 (entre 3.4 et $5.7 \mathrm{kmol}_{\mathrm{c}} /[\mathrm{ha} \times \mathrm{an}]$ sur le Plateau suisse dans Kurz et al 1998), en raison notamment des réductions massives des émissions de soufre entre 1985 et 1995. Les mesures des dépôts sur les sites LWF ont commencé en 1995 ou plus tard, c'est-à-dire après la période de réduction massive. Depuis le début des mesures, nous avons déterminé une diminution significative ( $\mathrm{p}<0.01)$ des dépôts acides sur trois sites (Jussy, Lausanne et Othmarsingen) uniquement (tableau 2). Les dépôts à Novaggio montrent une tendance décroissante mais peu significative. Les dépôts sur les autres sites sont restés stables.

Les dépôts de soufre ont diminué de manière significative $(\mathrm{p}<0.01)$ sur toutes les stations (tableau 2), ce qui reflète le déclin continu des émissions de soufre après le milieu des années 1990. Bien que les dépôts de soufre aient diminué partout, ils n'ont pas, ou peu, contribué à diminuer les dépôts acides compte tenu de leur faible proportion (environ $30 \%$ des dépôts acides). Les apports acides contiennent surtout de l'azote. Les retombées d'azote ont diminué de manière significative $(\mathrm{p}<0.01)$ sur trois (Jussy, Lausanne et Othmarsingen) des neuf sites étu- diés (voir Thimonier et al 2012, ce volume), conduisant ainsi à une diminution des dépôts acides (tableau 2). A Schänis, Beatenberg et Celerina, les dépôts d'azote tendent à diminuer, mais la baisse est peu significative pour l'instant (voir tableau 1 dans Thimonier et al 2012, ce volume). Les dépôts de cations basiques, importants pour neutraliser les apports d'azote et de soufre, sont restés constants en moyenne malgré de fortes variations annuelles (Graf Pannatier et al 2011).

Réactions de la chimie des solutions du sol Compte tenu de la diminution des retombées atmosphériques de soufre, on pourrait s'attendre à une diminution des concentrations de sulfate dans les solutions du sol, en particulier sous la litière. Nos mesures montrent effectivement que les concentrations de sulfate diminuent sous la litière $(0 \mathrm{~cm})$, voire à plus grande profondeur, dans la plupart des sites. A Vordemwald par exemple, nous avons observé une diminution marquée des concentrations de sulfate à toutes les profondeurs (tableau 3).

Afin de comparer les quantités de soufre arrivant et quittant l'écosystème forestier au cours du temps, nous avons comparé les apports annuels de sulfate provenant des pluviolessivats aux flux de sulfate quittant la litière $(0 \mathrm{~cm})$ et l'espace racinaire à $80 \mathrm{~cm}$ de profondeur depuis le début des mesures (figure 5). Les flux de sulfate sous la litière sont équivalents à ceux provenant des pluviolessivats et ont également tendance à diminuer au cours des années. Mais le déclin dans l'eau du sol n'est pas ou peu significatif dans la plupart des sites, compte tenu des larges variations annuelles des flux et de la période d'observation relativement courte (tableau 3). Seul à Vordemwald et à Beatenberg, les flux de sulfate sous la litière diminuent de manière significative ( $\mathrm{p}<0.01$ ). A $80 \mathrm{~cm}$ de profondeur, les flux de sulfate sont beaucoup plus élevés (de 6 à $37 \mathrm{~kg} \mathrm{S/(ha \times an)}$ que ceux estimés dans les pluviolessivats (de 3 à $17 \mathrm{~kg} \mathrm{~S} /[$ ha $\times$ an]) à Lausanne, Vordemwald et Novaggio, indiquant que le sol représente une source de sulfate (figure 5). L'excès de sulfate relâché dans la solution du sol provient probablement de sulfate, adsorbé aux hydroxydes d'aluminium et de fer pendant les années d'apports massif de soufre atmosphérique (Graf Pannatier et al 2011). Ce processus, rapporté dans plusieurs études en Europe, contribue à acidifier les eaux (p. ex. Alewell et al 2000). Dans les autres sites, les flux de sulfate à $80 \mathrm{~cm}$ sont du même ordre de grandeur que ceux mesurés dans les pluviolessivats. Les flux à $80 \mathrm{~cm}$ ont tendance à diminuer, mais compte tenu des larges variations an-

\footnotetext{
3 LIBISELLER C (2004) MULTMK/PARTMK, a program for the computation of Multivariate and Partial Mann-Kendall Test. www.slu.se $\rightarrow$ Fakulteten för naturresurser och lantbruksvetenskap $\rightarrow$ Om fakulteten $\rightarrow$ Institutioner $\rightarrow$ Institutionen för ekonomi $\rightarrow$ Personal $\rightarrow$ Partial Mann-Kendall tests (14.8.2012)
} 


\begin{tabular}{|c|c|c|c|c|c|c|c|c|c} 
& $\begin{array}{c}\text { Début des } \\
\text { mesures }\end{array}$ & $\begin{array}{c}\text { Profondeur } \\
(\mathrm{cm})\end{array}$ & $\begin{array}{c}\mathrm{Al} \\
(\mu \mathrm{mol} / \mathrm{I})\end{array}$ & $\begin{array}{c}\mathrm{BC} \\
(\mu \mathrm{mol} / \mathrm{I})\end{array}$ & $\begin{array}{c}\mathrm{BC} / \mathrm{Al} \\
(\mathrm{mol} / \mathrm{mol})\end{array}$ & $\begin{array}{c}\mathrm{N}_{\min } \\
(\mu \mathrm{mol} / \mathrm{I})\end{array}$ & $\begin{array}{c}\mathrm{SO}_{4} \\
(\mu \mathrm{mol} / \mathrm{I})\end{array}$ & $\begin{array}{c}\mathrm{Flux} \mathrm{SO}_{4} \\
\left(\mathrm{~kg} \mathrm{~S} \mathrm{SO}_{4} /\right. \\
{[\mathrm{ha} \times \mathrm{an}]}\end{array}$
\end{tabular}

Jura

\begin{tabular}{|c|c|c|c|c|c|c|c|c|}
\hline \multirow[t]{4}{*}{ Bettlachstock } & 2001 & 0 & $<$ LD & $=$ & n.d. & $=$ & $(\downarrow)$ & $(\Downarrow)$ \\
\hline & & 15 & $<\mathrm{LD}$ & $=$ & n.d. & $=$ & $=$ & $=$ \\
\hline & & 50 & $<\mathrm{LD}$ & $=$ & n.d. & $=$ & $=$ & $=$ \\
\hline & & 80 & $<$ LD & $=$ & n.d. & $=$ & $=$ & $=$ \\
\hline
\end{tabular}

\section{Plateau suisse}

\begin{tabular}{|l|c|c|c|c|}
\hline Lausanne & 2000 & 0 & $=$ & \\
\hline & & 15 & $=$ & \\
\hline & & 50 & $=$ & \\
\hline Vordemwald & 2000 & 0 & $(\downarrow)$ & \\
& & 15 & $\downarrow \downarrow$ & $\downarrow$ \\
\hline & & 50 & $\downarrow \downarrow$ & $\downarrow$ \\
\hline & & 80 & $=$ & $\downarrow$ \\
\hline
\end{tabular}

\begin{tabular}{c|c|c|}
$=$ & $=$ \\
$=$ & $=$ \\
$=$ & $=$ \\
\hline$=$ & $(\downarrow)$ \\
\hline$\downarrow$ & $=$ & \\
\hline$\downarrow \downarrow$ & $\downarrow$ & $(\downarrow)$ \\
\hline$\downarrow \downarrow$ & $=$ & \\
\hline$\downarrow \downarrow$ & $(\downarrow)$ & \\
\hline
\end{tabular}

\begin{tabular}{c|c|c|}
$=$ & $=$ & $=$ \\
$=$ & $=$ & $=$ \\
\hline$=$ & $(\downarrow)$ & $(\downarrow)$ \\
$=$ & $=$ & $\downarrow$ \\
$=$ & $\downarrow$ & $\downarrow$ \\
\hline$(\downarrow)$ & $\downarrow \downarrow$ & $(\downarrow)$ \\
\hline$\downarrow$ & $\downarrow \downarrow$ & $(\downarrow)$ \\
\hline$\downarrow$ & $\downarrow \downarrow$ &
\end{tabular}

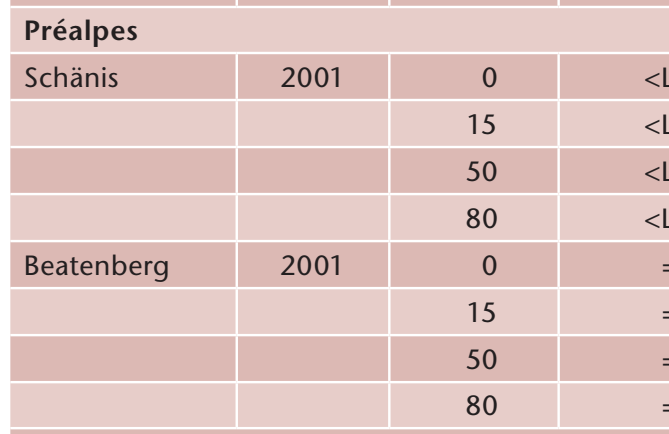

\begin{tabular}{l|l|}
$<\mathrm{LD}$ \\
$<\mathrm{LD}$ \\
$<\mathrm{LD}$ \\
$<\mathrm{LD}$ \\
$=$ \\
$=$ \\
$=$ \\
$=$
\end{tabular}

\begin{tabular}{|c|}
\hline$=$ \\
\hline$\downarrow$ \\
\hline$=$ \\
\hline$=$ \\
\hline$=$ \\
\hline$\downarrow$ \\
\hline$=$ \\
\hline$=$ \\
\hline
\end{tabular}

\begin{tabular}{|c|c|c|}
\hline n.d. & $=$ & $(\downarrow)$ \\
\hline n.d. & $=$ & $(\downarrow)$ \\
\hline n.d. & $=$ & $\downarrow$ \\
\hline n.d. & $=$ & \\
\hline$(\Downarrow)$ & $=$ & $(\downarrow$ \\
\hline$\downarrow$ & $=$ & $\downarrow$ \\
\hline$=$ & $(\downarrow)$ & $(\downarrow)$ \\
\hline$=$ & $=$ & $=$ \\
\hline
\end{tabular}

\begin{tabular}{|c|c|}
\hline$(\downarrow)$ & $=$ \\
\hline$(\downarrow)$ & $(\downarrow)$ \\
\hline & $\downarrow$ \\
$\downarrow$ & $(\downarrow)$ \\
\hline$(\downarrow)$ & $\Downarrow$ \\
$\downarrow$ & $\downarrow$ \\
\hline$(\downarrow)$ & $(\downarrow)$ \\
\hline$=$ & $=$
\end{tabular}

\section{Alpes}

\begin{tabular}{|l|r|r|}
\hline Celerina & 2001 & 0 \\
\hline & & 15 \\
\hline & 50 \\
\hline & & 80 \\
\hline
\end{tabular}

\begin{tabular}{ll|l|}
$=$ & $=$ \\
$=$ & $=$ \\
$=$ & $=$ \\
$=$ & $=$
\end{tabular}

\begin{tabular}{c|c|c|c}
$=$ & $=$ & $=$ & $(\boldsymbol{\Downarrow})$ \\
$=$ & $<\mathrm{LD}$ & $=$ & $=$ \\
$=$ & $<\mathrm{LD}$ & $=$ & $=$ \\
$=$ & $<\mathrm{LD}$ & $=$ & $=$
\end{tabular}

\section{Tessin}

\begin{tabular}{|l|c|c|c|c|c|}
\hline Novaggio & 2001 & 0 & $(\boldsymbol{})$ & $=$ \\
\hline & & 15 & $=$ & $=$ \\
\hline & & 50 & $=$ & $=$ \\
\hline Copera & & 80 & $=$ & $=$ \\
\hline & 2000 & 0 & $=$ & $\downarrow$ \\
\hline & 30 & $(\boldsymbol{\Downarrow})$ & $\downarrow$ \\
\hline & & 57 & $(\boldsymbol{\Downarrow})$ & $(\boldsymbol{\Downarrow})$ \\
\hline
\end{tabular}

\begin{tabular}{|l|l|}
\hline$\downarrow$ & $=$ \\
\hline$=$ & $=$ \\
\hline$=$ & $=$ \\
\hline$=$ & \\
\hline$(\downarrow)$ & \\
\hline$=$ & $=$ \\
$=$ & $=$ \\
\hline$=$ & $=$ \\
\hline
\end{tabular}

\begin{tabular}{l|c|c|}
$=$ & $(\Downarrow)$ & $(\Downarrow)$ \\
$=$ & $(\Downarrow)$ & $=$ \\
$=$ & $\downarrow$ & $=$ \\
$=$ & $(\Downarrow)$ & $=$ \\
$\downarrow$ & $\Downarrow$ & n.d. \\
$=$ & $=$ & n.d. \\
$=$ & $(\Downarrow)$ & n.d. \\
$=$ & $=$ & n.d.
\end{tabular}

Tab. 3 Tendances temporelles des concentrations d'aluminium ( $A l)$, de cations basiques $(B C)$, d'azote minéral $\left(\mathrm{N}_{\text {min }}\right)$, de sulfate $\left(\mathrm{SO}_{4}\right)$, du rapport $B C / A l$ et des flux de sulfates dans les solutions du sol à quatre profondeurs. = pas de tendance; $(\downarrow)$ diminution peu significative $(p<0.05)$; $\downarrow$ diminution significative $(p<0.01)$, $\downarrow \downarrow$ diminution très significative $(p<0.001)$. $<$ LD: plus petit que la limite de détection, $n . d .:$ non déterminé.

nuelles, cette diminution n'est pas ou peu significative (tableau 3).

Nous avons suivi l'évolution temporelle du rapport $\mathrm{BC} / \mathrm{Al}$ dans les sols acides (Lausanne, Vordemwald, Beatenberg, Celerina, Novaggio et Copera) à quatre profondeurs, pendant toute la période d'observation (figure 6). Les rapports BC/Al sont restés stables à Lausanne et Celerina, suggérant que l'acidification y est très lente (pas visible en dix ans) et qu'il n'y a pas eu de détérioration du taux de saturation du sol. Par contre, une diminution significative de $\mathrm{BC} / \mathrm{Al}$ a été rapportée à $15 \mathrm{~cm}$ de profondeur à Vordemwald et Beatenberg, suggérant une acidifica- tion rapide de l'eau du sol (visible en 10 ans). Une diminution du rapport BC/Al a été observée sous la litière au Tessin à Novaggio et Copera (tableau 3). Cependant, celle-ci est due à une augmentation passagère du pH entre 2000 et 2001, causant une diminution marquée des concentrations d'aluminium dans la solution du sol au début de la période d'observation. Des apports importants de cations basiques transportés dans les poussières du Sahara pourraient être à l'origine de cette augmentation du pH (Rogora et al 2004). Dans les horizons minéraux à Copera, une forte diminution des rapports BC/Al fut observée entre 1987 et 1997, indiquant une aci- 

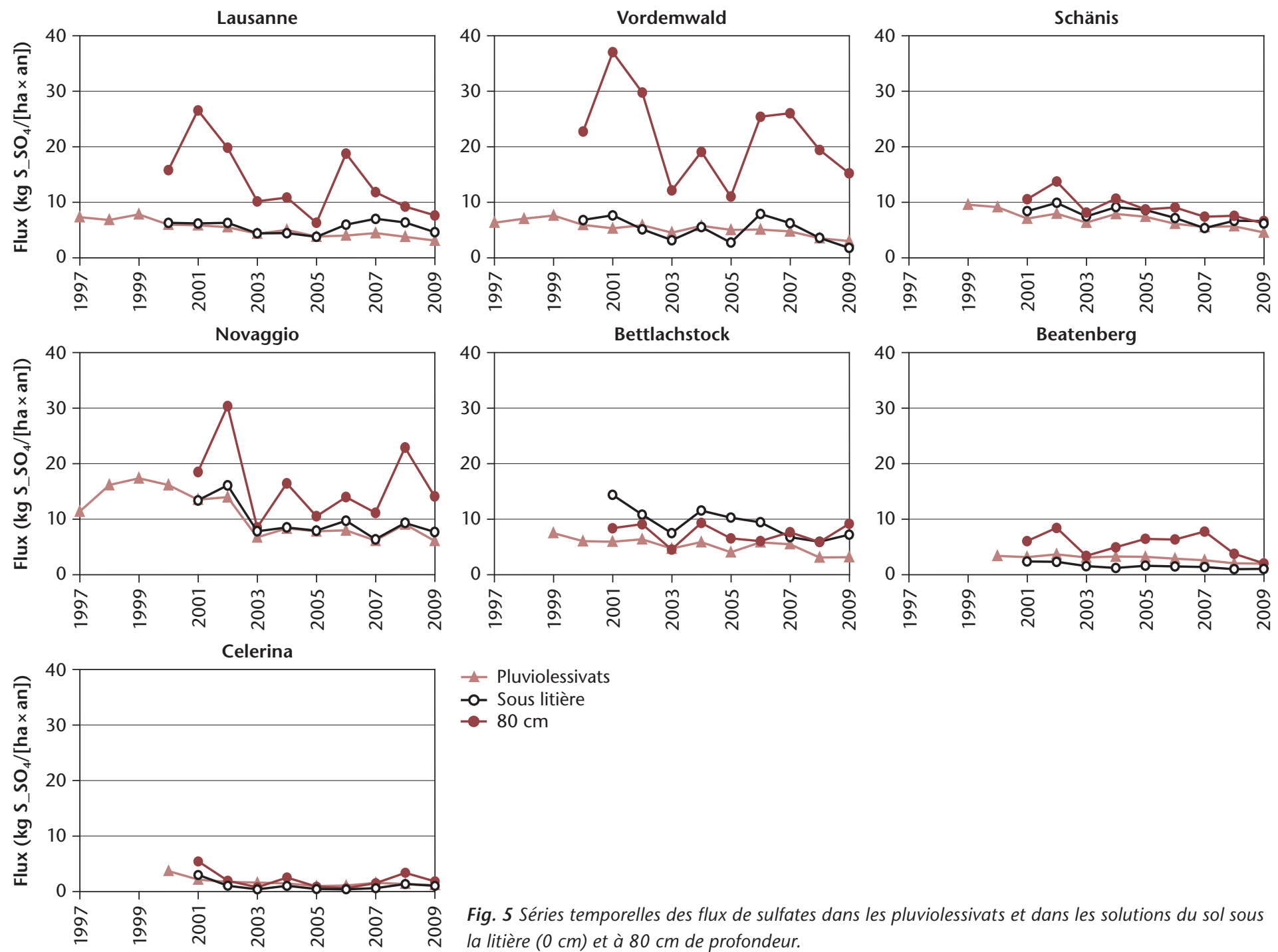

- Pluviolessivats
-o- Sous litière

$\rightarrow 80 \mathrm{~cm}$

Fig. 5 Séries temporelles des flux de sulfates dans les pluviolessivats et dans les solutions du sol sous la litière $(0 \mathrm{~cm})$ et à $80 \mathrm{~cm}$ de profondeur.

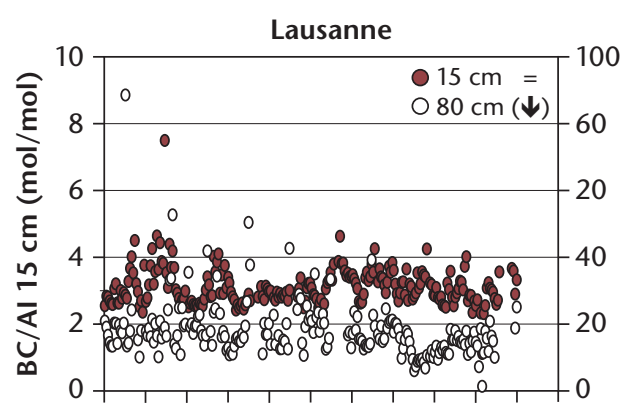

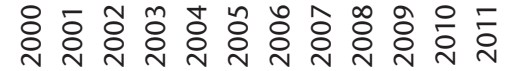

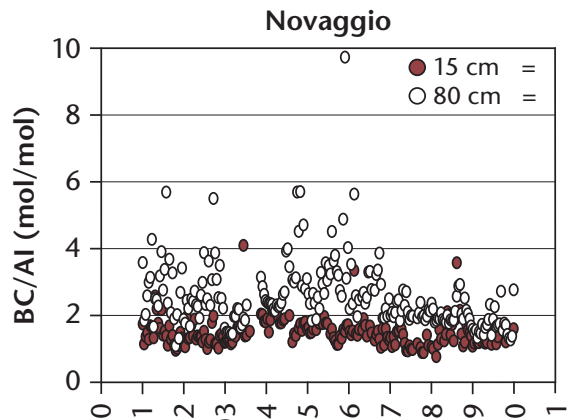

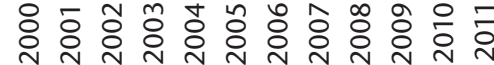

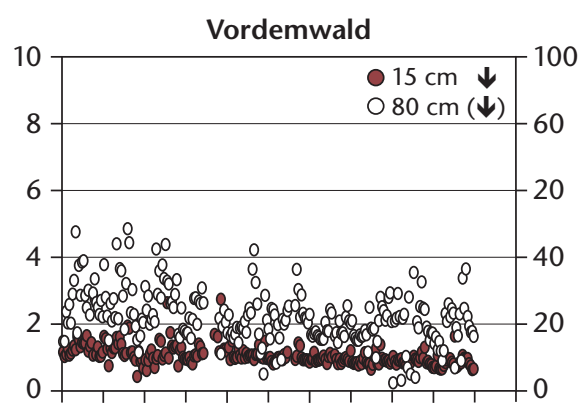

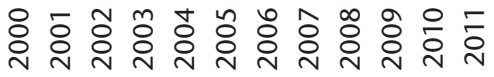

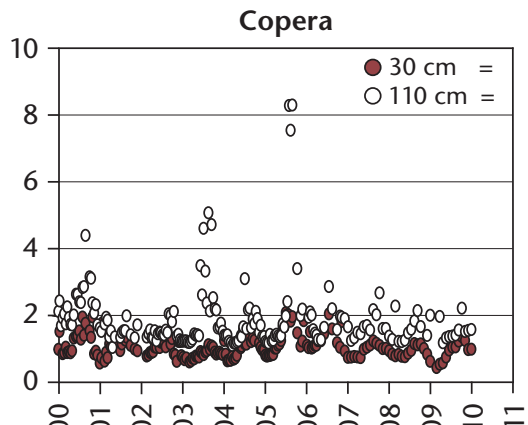

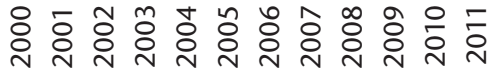

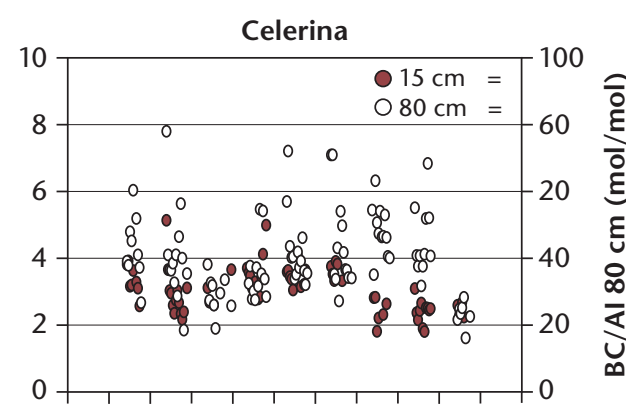

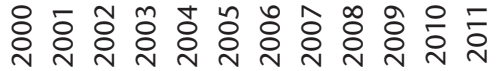

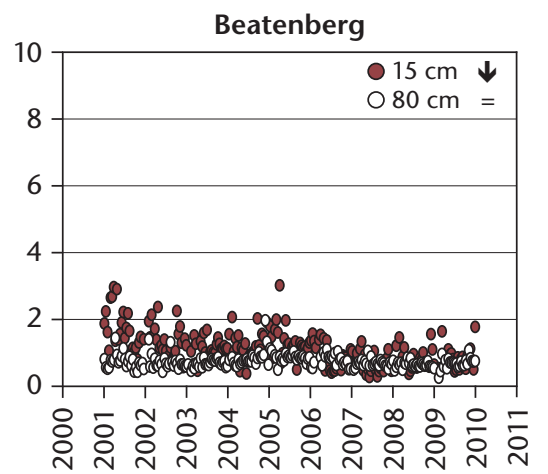

Fig. 6 Séries temporelles des rapports BC/Al (mol/mol) dans les solutions du sol à $15 \mathrm{~cm}$ et $80 \mathrm{~cm}$ de profondeur depuis 2000 (ou plus tard) jusqu'en 2009 . A Lausanne, Vordemwald et Celerina, l'échelle des rapports $B C / A l \grave{a} 80 \mathrm{~cm}$ de profondeur se trouve sur l'axe à droite du graphique. Les tendances temporelles (voir tableau 2) sont indiquées dans la légende: = pas de tendance; $(\Downarrow)$ diminution peu significative $(p<0.05)$; $\Downarrow$ diminution significative $(p<0.01)$. 


\begin{tabular}{|c|c|c|c|c|c|c|}
\hline Risque & $\begin{array}{l}\text { Elevé } \\
(\mathrm{n})\end{array}$ & $\begin{array}{l}\text { Modéré } \\
\text { (n) }\end{array}$ & $\begin{array}{l}\text { Aucun } \\
\text { (n) }\end{array}$ & $\begin{array}{l}\text { Elevé } \\
(\%)\end{array}$ & $\begin{array}{c}\text { Modéré } \\
(\%)\end{array}$ & $\begin{array}{c}\text { Aucun } \\
(\%)\end{array}$ \\
\hline \multicolumn{7}{|l|}{ Type de sol } \\
\hline Sols bruns (para-, podzolique, etc.) & 132 & 127 & 235 & 27 & 26 & 48 \\
\hline Podzols (crypto-, humo-ferrugineux, etc.) & 48 & 20 & 12 & 60 & 25 & 15 \\
\hline Pseudogleys & 9 & 13 & 7 & 31 & 45 & 24 \\
\hline Stagnogleys & 6 & 8 & 1 & 40 & 53 & 7 \\
\hline Rendzines (para-, brunifiée, etc.) & 0 & 0 & 321 & 0 & 0 & 100 \\
\hline Gleys & 0 & 3 & 63 & 0 & 5 & 95 \\
\hline Regosols & 0 & 2 & 14 & 0 & 13 & 88 \\
\hline Autres & 2 & 4 & 12 & 11 & 22 & 67 \\
\hline \multicolumn{7}{|l|}{ Total } \\
\hline \multicolumn{7}{|l|}{ Type d'humus } \\
\hline Mor & 73 & 42 & 32 & 50 & 29 & 22 \\
\hline Moder & 97 & 80 & 103 & 35 & 29 & 37 \\
\hline Mull & 27 & 52 & 501 & 5 & 9 & 86 \\
\hline Autres & 0 & 3 & 29 & 0 & 9 & 91 \\
\hline Total & & & & & & \\
\hline
\end{tabular}

Tab. 4 Répartition des 1039 profils de sol (nombre $n$ de profils et pourcentage) selon les types de sol et d'humus (classé selon Walthert et al 2004) dans chaque classe de risque d'acidification de l'eau du sol (élevé: taux de saturation TS moyen $<15 \%$; modéré: TS minimum $<15 \%$ mais TS moyen $\geq 15 \%$; aucun: TS minimum et TS moyen $\geq 15 \%$ ).

dification accélérée de l'eau du sol (Blaser et al 1999). Les rapports se sont stabilisés à la fin des années 1990 (Graf Pannatier et al 2005) et sont restés constants jusqu'en 2009 (tableau 3 et figure 6), suggérant un ralentissement de l'acidification.

\section{Distribution des sols à complexe désaturé}

La figure 1 illustre la distribution des 1039 profils de sol selon le risque d'acidification de la solution du sol à travers la Suisse. 19\% $(n=197)$ ont un taux de saturation (TS) moyen $<15 \%$ entre la surface du sol (premier horizon minéral) et $80 \mathrm{~cm}$ de profondeur. Nous considérons qu'il y a un risque élevé que l'eau du sol s'acidifie rapidement (diminution du rapport $\mathrm{BC} / \mathrm{Al}$ ) dans ces sols car les apports d'acides, qu'ils soient d'origine atmosphérique ou produits dans le sol, causent une mobilisation de l'aluminium dans la solution du sol. Les sols qui ont un TS minimum $<15 \%$ dans le profil mais en moyenne $\geq 15 \%$ représentent $17 \%(n=177)$ du collectif. Nous estimons qu'il y a un risque modéré d'acidification de la solution du sol car seule une partie du profil paraît critique. Ces profils présentent généralement un fort gradient du TS avec la profondeur, avec un minimum dans la partie supérieure du sol minéral $(10-30 \mathrm{~cm})$ et une augmentation du TS avec la profondeur. Les 665 autres profils (64\%) ont un TS minimum et moyen $\geq 15 \%$ et ne présentent pas de risque immédiat d'acidification de l'eau du sol.

Cette classification permet d'identifier les sols à risque, mais il n'y a pas nécessairement une acidification rapide de l'eau du sol à ces sites aujourd'hui. En effet, nous n'avons pas observé de diminution du rapport $\mathrm{BC} / \mathrm{Al}$ dans la solution du sol depuis le dé- but des années 2000 à Lausanne, Novaggio et Copera, bien que nous ayons classé ces profils dans la catégorie à risque élevé (tableau 1). Par contre à Beatenberg et à Vordemwald, également classés comme profils à risque élevé, nous avons détecté une diminution significative des rapports BC/Al dans la solution du sol.

Les profils à risque élevé (TS moyen <15\%) se trouvent au Tessin dans les sols développés à partir d'un substrat acide (roches cristallines), près de Disentis dans la vallée du Rhin antérieur (massif de Tavetsch), sur le Plateau dans les moraines du Riss et les grès molassiques. Dans les Préalpes et les Alpes, on les trouve dans les poudingues riches en silicates et les roches acides (grès, granite, gneiss) des massifs cristallins et des nappes alpines (helvétique, pennique). Parmi ces profils à complexe désaturé, on y trouve différents types de sol comme des podzols, des pseudo- et stagnogleys et des sols bruns (tableau 4). L'eau infiltrée dans les podzols s'acidifie rapidement (surtout à cause de la forte production d'acides organiques). En effet, 85\% des podzols $(n=80)$ se trouvent dans les classes de risque élevé et modéré (tableau 4). La décroissance du rapport $\mathrm{BC} / \mathrm{Al}$ dans la solution du sol à Beatenberg confirme d'ailleurs cette hypothèse. Les autres types de sol (les sols bruns en particulier) ne sont pas indicateurs d'un risque élevé d'acidification car on les retrouve dans toutes les classes de risques, compte tenu de la large gamme de valeurs du TS dans ces sols. Les types de sol ne présentant aucun risque d'acidification sont principalement les rendzines (tableau 4). Les gleys, sols influencés par la nappe phréatique, ont également un TS élevé et ne présentent aucun risque. 
Le type d'humus représente un paramètre utile pour prédire le risque d'acidification dans le profil. Le risque d'acidification de la solution du sol est plus élevé dans les sols avec un mor, et dans une moindre mesure avec un moder, que dans les sols couverts d'un mull (tableau 4). Ces derniers ne présentent dans la plupart des cas (91\%) aucun risque d'acidification. Les données de la solution du sol confirment cette hypothèse car les deux sols présentant une diminution du rapport $\mathrm{BC} / \mathrm{Al}$ sont caractérisés par un mor à Beatenberg et un moder à Vordemwald. Cependant, le rapport BC/Al n'a pas diminué à Novaggio (moder).

Pour la première fois, nous produisons une carte des risques (figure 1) à partir de données qui proviennent d'un nombre élevé de profils de sol (1039) répartis dans toute la Suisse. Elle donne une bonne vue d'ensemble sur la distribution des sols à complexe désaturé dans les forêts suisses. Cependant, les sols développés à partir de roches cristallines acides dans les Alpes sont sous-représentés et de ce fait, la proportion de sol présentant un risque d'acidification est probablement sous-estimée dans cette région.

\section{Conclusions}

L'analyse des données récoltées dans le projet LWF nous permet de répondre aux trois questions posées dans l'introduction: 1) les dépôts atmosphériques acides n'ont diminué de manière significative que dans trois des neufs stations analysées entre 1995 (ou plus tard selon la date d'installation) et 2009 . Bien que les dépôts de soufre aient diminué sur tous les sites, les apports d'azote restent élevés, maintenant ainsi le même niveau d'apports acides. 2) La réduction des dépôts atmosphériques de soufre se reflète dans la solution du sol sous la litière dans la plupart des sites étudiés. Par contre à $80 \mathrm{~cm}$ de profondeur, le déclin peut être retardé par la désorption de sulfates accumulés dans le sol. Dans trois des sept sites analysés, le sol relâche chaque année de grande quantité de sulfates accompagnés de cations basiques, contribuant ainsi à appauvrir le sol en nutriments. De manière générale, la composition chimique des solutions du sol aux sept sites LWF et à Copera a relativement peu changé depuis le début des années 2000. Parmi les cinq sites dont le sol est sensible à l'acidification (Lausanne, Novaggio, Beatenberg, Vordemwald, Copera), les rapports $\mathrm{BC} / \mathrm{Al}$ sont restés stables dans trois d'entre eux (Lausanne, Novaggio, Copera), alors qu'à Vordemwald et Beatenberg, la diminution de BC/Al témoigne d'une rapide acidification de l'eau du sol. 3) Le taux moyen de saturation entre 0 et $80 \mathrm{~cm}$ de profondeur dans 1039 profils de sol à travers la Suisse nous montre qu'il y a un risque élevé (TS moyen <15\%) d'acidification de l'eau du sol dans près de $20 \%$ des profils. Cependant, la propor- tion des sites où l'acidification se produit effectivement de manière accélérée est probablement plus faible. L'analyse temporelle des rapports $\mathrm{BC} / \mathrm{Al}$ dans les solutions du sol montre qu'à quantité égale de dépôts acides, les vitesses d'acidification dans les sols à complexe désaturé (<15\%) diffèrent d'un site à l'autre.

Soumis: 2 décembre 2011, accepté (avec comité de lecture): 30 juillet 2012

\section{Références}

ALEWELL C, MANDERSCHEID B, MEESENBURG H, BITTERSOHL J (2000) Is acidification still an ecological threat? Nature 407: 856-857.

ALEWELL C ET AL (2001) Are there signs of acidification reversal in freshwaters of the low mountain ranges in Germany? Hydrol Earth Syst Sci 5: 367-378.

ARMBRUSTER M, ABIY M, FEGER KH (2003) The biogeochemistry of two forested catchments in the Black Forest and the eastern Ore Mountains (Germany). Biogeochem 65: 341-368.

BLASER P, ZYSSET M, ZIMMERMANN S, LUSTER J (1999) Soil acidification in southern Switzerland between 1987 and 1997: a case study based on the critical load concept. Environ Sci Technol 33: 2383-2389.

BLASER P, WALTHERT L, GRAF PANNATIER E (2008) The sensitivity of forest soils to acidification and the risk of aluminium toxicity. J Plant Nutr Soil Sci 171: 605-612.

EVANS CD ET AL (2001) Recovery from acidification in European surface waters. Hydrol Earth Syst Sci 5: 283-297.

FAO (1988) FAO/Unesco Soil Map of the World, Revised Legend, with corrections and updates. Rome: FAO, World Soil Resources Report 60.

GRAF PANNATIER E, LUSTER J, ZIMMERMANN S, BLASER P (2005) Acidification of soil solution in a chestnut forest stand in southern Switzerland: are there signs of recovery? Environ Sci Technol 39: 7761-7767.

GRAF PANNATIER E, THIMONIER A, SCHMITT M, WALTHERT L, WALDNER P (2011) A decade of monitoring at Swiss LongTerm Forest Ecosystem Research (LWF) sites: can we observe trends in atmospheric acid deposition and in soil solution acidity? Environ Monit Assess 174: 3-30.

HILDEBRAND EE, VON WILPERT K, BUBERL HG (1996) Erkenntnismöglichkeiten an Waldökosystemen im Spannungsfeld zwischen grossräumiger Mustererkennung und dem «eisernen Gesetz des Örtlichen». Allg Forst- Jagdztg 167: 9-10.

HIRSCH RM, SLACK JR (1984) A nonparametric trend test for seasonal data with serial dependence. Water Resour Res 20: 727 732.

JANSSON PE, KARLBERG L (2004) Coupled heat and mass transfer model for soil-plant-atmosphere systems. Stockholm: Royal Institute Technology, Dept Civil Environmental Engineering. 435 p. ftp://www.Iwr.kth.se/CoupModel/CoupModel.pdf (31.7.2012)

KURZ D, RIHM, B, SVERDRUP H, WARFVINGE P (1998) Critical loads of acidity for forest soils. Regionalized PROFILE Model. Berne: Swiss Agency Environment Forests Landscape, Environmental Documentation 88. $115 \mathrm{p}$.

LIBISELLER C, GRIMVALL A (2002) Performance of partial MannKendall tests for trend detection in the presence of covariates. Environmetrics 13: 71-84.•

IKENS GE ET AL (1998) The biogeochemistry of calcium at Hubbard Brook. Biogeochemistry 41: 89-173.

OFEV (2009) NABEL - La pollution de l'air 2008. Mesures exécutées à I'aide du Réseau national d'observation des polluants atmosphériques (NABEL). Berne: Office fédéral de l'environnement, Etat de l'environnement 0919. 139 p. 
ROGORA M, MOSELLO R, MARCHETTO A (2004) Long-term trends in the chemistry of atmospheric deposition in Northwestern Italy: the role of increasing Saharan dust deposition. Tellus 56B: 426-434.

SCHÖPP W, POSCH M, MYLONA S, JOHANSSON M (2003) Longterm development of acid deposition (1880-2030) in sensitive freshwater regions in Europe. Hydrol Earth Syst Sci 7: 436446.

STODDARD JL ET AL (1999) Regional trends in aquatic recovery from acidification in North America and Europe. Nature 401: 575-57

SVERDRUP H, WARFVINGE P (1993) The effect of soil acidification on the growth of trees, grasses and herbs as expressed by the $(\mathrm{C} a+\mathrm{Mg}+\mathrm{K}) / \mathrm{Al}$ ratio. Lund: Lund Univ, Depart Chemical Engin, Rep Ecol Environm Engin 2. 108 p.

THIMONIER A ET AL (2012) Dépôts atmosphériques azotés et leurs effets en forêt: un bilan des sites d'observation à long terme. Schweiz Z Forstwes 163: 343-354. doi: 10.3188/szf.2012.0343

THIMONIER A, SCHMITT M, WALDNER P, RIHM B (2005) Atmospheric deposition on Swiss long-term forest ecosystem research (LWF) plots. Environ Monit Assess 104: 81-118.
ULRICH B (1983) Interaction of forest canopies with atmospheric constituents: $\mathrm{SO}_{2}$, Alkali and Earth Alkali Cations and Chloride. In: Ulrich B, Pankrath J, editors. Effects of accumulation of air pollutants in forest ecosystems. Dordrecht: Reidel. pp. 33-45.

ULRICH B (1991) An ecosystem approach to soil acidification. In: Ulrich B, Sumner ME, editors. Soil acidity. Berlin: Springer. pp. 28-79.

UNECE (2004) Manual on methodologies and criteria for modelling and mapping critical loads and levels and air pollution effects, risks and trends. ftp://ftp.rivm.nl/cce/outgoing/Web/ pdfs/Manual/Manual\%20UBA\%20Texte.pdf (31.7.2012)

WALTHERT L, BLASER P, LÜSCHER P, LUSTER J, ZIMMERMANN S (2003) Langfristige Waldökosystem-Forschung LWF. Kernprojekt Bodenmatrix. Ergebnisse der ersten Erhebung 1994-1999. doi: 10.3929/ethz-a-004500705

WALTHERT L, ZIMMERMANN S, BLASER P, LUSTER J, LÜSCHER P (2004) Waldböden der Schweiz. Band 1. Grundlagen und Region Jura. Bern: HEP. 768 p.

WATMOUGH SA, DILLON PD (2004) Major element fluxes from a coniferous catchment in central Ontario, 1983-1999. Biogeochemistry 67: 369-398.

\section{Impacts des dépôts atmosphériques acides sur l'eau des sols forestiers}

Après un apport massif de substances acidifiantes sur l'environnement au milieu du XXe siècle, les retombées d'acides ont diminué grâce aux réductions d'émissions de soufre. L'apport continu des dépôts acides peut modifier la chimie des sols et des eaux d'infiltration, et accélérer leur acidification naturelle. Dans le cadre du projet de recherches à long terme sur les écosystèmes forestiers (LWF), nous avons examiné si les apports atmosphériques acides ont continué à décroître ces dix dernières années dans différents écosystèmes forestiers et comment la chimie de l'eau du sol a réagi à cette amélioration de la qualité de l'air. Les retombées acides n'ont diminué de manière significative que sur trois des neuf sites analysés. Les dépôts de soufre ont diminué partout, mais comme ils constituent une fraction mineure par rapport aux dépôts d'azote, ils n'ont pas contribué à diminuer les dépôts acides. La chimie des solutions du sol a relativement peu changé depuis une dizaine d'années. Nous n'avons pas observé d'acidification de l'eau du sol dans six des huit sites analysés. Par contre, les changements de la chimie de l'eau du sol dans deux stations témoignent d'une rapide acidification. Dans trois sites, le sol $(50-80 \mathrm{~cm})$ a relâché de grande quantité de sulfate accompagné de cations basiques, contribuant probablement à appauvrir le sol en nutriments. L'analyse du taux de saturation de 1039 profils de sol à travers la Suisse montre qu'il y a un risque élevé d'une acidification de l'eau du sol dans près de $20 \%$ des profils.

\section{Auswirkungen saurer atmosphärischer Einträge auf die Bodenlösung im Wald}

In der Mitte des 20. Jahrhunderts war die Umwelt massiven versauernden Stoffeinträgen ausgesetzt. Danach nahm die saure atmosphärische Deposition aufgrund reduzierter Schwefelemissionen ab. Kontinuierliche Säureeinträge können die chemische Beschaffenheit der Böden und des Sickerwassers beeinflussen und die Bodenversauerung beschleunigen. Im Rahmen der Langfristigen Waldökosystem-Forschung (LWF) untersuchten wir in verschiedenen Waldökosystemen, ob die saure Deposition in den vergangenen zehn Jahren weiter abgenommen hat und wie die Chemie des Bodenwassers auf die verbesserte Luftqualität reagiert hat. Die saure Deposition hat nur in drei von insgesamt neun Untersuchungsflächen abgenommen. Zwar wurde auf allen Untersuchungsflächen weniger Schwefel abgelagert. Da sein Anteil im Vergleich zum Stickstoff gering ist, wirkte sich der verminderte Schwefeleintrag kaum auf die saure Deposition aus. Seit Messbeginn vor rund zehn Jahren veränderte sich die Chemie der Bodenlösung nur wenig. In sechs von acht Böden wurde keine Versauerung der Bodenlösung beobachtet. Auf zwei Untersuchungsflächen dagegen deuten Veränderungen in der Chemie der Bodenlösung auf eine schnelle Versauerung hin. Auf drei Untersuchungsflächen wurden grosse Mengen an Sulfat gekoppelt mit basischen Kationen aus dem Unterboden ausgewaschen, was zur Nährstoffverarmung dieser Böden beitrug. Die Analyse der Basensättigung von 1039 über die Schweiz verteilten Waldbodenprofilen ergab, dass bei nahezu $20 \%$ dieser Böden ein hohes Risiko für eine relativ rasche Versauerung der Bodenlösung besteht. 